\title{
IMPROVEMENTS FOR DMM IN SDN AND VIRTUALIZATION-BASED MOBILE NETWORK ARCHITECTURE
}

\author{
Muhammad Dawood ${ }^{1,2}$, Woldemar Fuhrmann ${ }^{1,2}$, and Bogdan V Ghita ${ }^{1}$ \\ ${ }^{1}$ Centre for Security, Communications and Network Research, Plymouth University UK \\ ${ }^{2}$ Faculty of Computer Science, University of Applied Sciences Darmstadt, Germany
}

\begin{abstract}
The (r)evolution of wireless access infrastructure can be described as the convergence of the available radio communication systems towards a harmonized, more flexible and reconfigurable access system to match the current and upcoming demands. In recent years Softwarization and Virtualization technologies have moved from server and network domains to wireless domain and provides new perspectives of managing mobile networks functionalities. This paper provides evolution of the mobile network architecture in Software Defined Networking (SDN) and virtualization context and realizes it through the use of distribution of gateway function approach. Key improvements with proposed approach are to support efficient mobility management in heterogeneous access environments, remove the chains of IP preservation and optimal data path management according to application needs. A functional setup validates and assays the proposed evolution in terms of inter-system handover preparation, interruption and completion time relative to control plane delay requirements of the $5 G$ networks.
\end{abstract}

\section{KEYWORDS}

Software Defined Mobile Network; Network Functions Virtualization; Inter-System Inter-networking; SDN based DMM; IP mobility management

\section{INTRODUCTION}

The rapid growth of mobile data traffic has been widely recognized and reported and the mobile communications industry is preparing to cope with a 1000x increase of traffic by 2020 over 2010 [1]. More and more people see their handheld devices as an annex of their workspace while on move and the continuous improvement to the mobile network architecture is becoming increasingly important to support the performance requirements for the ubiquitous wireless connections. Specifically to support different levels of Mobile Unit (MU) mobility, future wireless access infrastructure is required to a) Support integration of heterogeneous Radio Access Technologies (RATs), the 3GPP Long Term Evolution (LTE) [2] and trusted/untrusted non-3GPP access types (for example Wireless LAN) efficiently combining multiple simultaneous connections of MU via multiple access nodes with unified mobility management b) provide seamless IP mobility for session as well as service continuity as per application needs. c) maintaining optimal service level quality for services that have different latency requirements between the MU and the Packet Data Network (PDN); and d) Support optimized mechanisms to control signaling overhead i.e. Minimize the number of messages required to enable the traffic exchange between the MU and the PDN.

Current and upcoming wireless communication systems exploit many techniques to meet these requirements. Wireless Local Area Networks (WLANs) provide high data rates at low cost within a limited area. Cellular systems serve wide coverage areas, full mobility and roaming, combining multiple radio resources and deploying a mix of different radio access networks (RANs), frequencies, cell sizes with new ways of acquiring, deploying, operating and managing multiple DOI : 10.5121 /ijmnct.2018.8401 
(RATs). However, the current mobile network architecture is highly hierarchical and centralized, several limitations have been identified when compared to the always-on network access service requirements of seamless mobility. With centralized network architecture, since the MU uses the single address anchored at central IP anchor, user traffic will always need to go first to the home network and then to the correspondent service node even it is not connected via home access network, leading to paths that are in general longer than the direct one between the MU and its correspondent service node. This poses excessive traffic concentration on a single gateway element and possibly un-optimized routing adding in turn unnecessary delay and wasting resources. Centralized solutions are probable to have reliability problem, as the central entity is potentially a single point of failure. Central IP mobility anchor have to deal with higher user traffic simultaneously, thus need to have enough processing and routing capabilities implies several scalability and network design problems [3]. To cope with the problems of centralized mobility management: in a previous work [4] we followed the concept of distributed mobility management (DMM) in contrast to centralized approach in a hierarchical model. The basic concept of DMM is the distribution of mobility and IP anchors and select and re-locate when necessary gateways that are topologically/geographically close to the MU. DMM architecture solves some of the problems of centralized mobility management, however when a MU moves to a new set of anchors, due to IP relocation, either tunneling need to be used between initial router and new router or the active flows have to maintained until the flow is finished. Consequently, it may lead to a complex process and a high signaling cost.

Further more. due to the growing demand of wireless communications for a wide range of purposes, wireless network infrastructure requires more flexibly and to make them adaptive to match the actual demands. In this context during the recent years Software Defined Networking (SDN) and Virtualization of network functionalities have gained a lot of attention from the research community and standardization organizations [5-6]. SDN provides flexible ways to monitor and manage network efficiently with separation of data plane and control plane. Virtualization allows the hardware infrastructure to be provided as a service by abstraction and sharing of physical resources. Both technologies have related mechanisms and therefore complement one another.

In line with these trends we set out to take up the current mobile network architecture what we refer as Software defined plus virtualization featured Mobile Network (S+ MN), combined with programmable software instances capable of running on general purpose servers via virtualization technologies to solve many of issues described above. To limit the potentially huge design space and the envisioned research towards distribution of gateway functions we focus mainly on seamless mobility in considered scenario, which include maintaining QoS, smooth handover, session continuity and sharing across multiple wireless technologies. Although there have been several proposals in this field as surveyed in [7-10] but these studies have only proposed that SDN and virtualization of network functions can provide much-needed benefits but have not shown the details for the evolved architecture. This paper not only proposed the S+ MN Architecture but also provide realization of core network functions with details of procedure commonly occur in current mobile networks elaborated with the separation of data and control plane. To summarize, the main contributions of this paper can be stated as follows:

- Improvements for Software defined Plus virtualization featured Mobile Network (S+ MN) architecture with details of functional elements.

- Description of the procedures of current mobile networks to SDN and wireless virtualization context using the proposed S Plus controllers; Initial attachment, service setup and QoS flow marking, inter-system handover and the data plane management in a distributed manner, etc. 
- By analysing current research of IP mobility management; the proposed architecture implied Multipath TCP (MPTCP) functions to remove the chains of IP address preservation for session continuity during IP anchor relocation without the use of bidirectional tunnels between the initial and new router and avoiding a large number of signalling messages.

- Development of a functional validation setup to show the working of S+ MN architecture, application oriented forwarding of IP traffic through the use of distributed Data Plane Entities (DPEs) and select the right one for use in the following cases. i) The MU acts as a server and requires a static IP address for incoming IP flows. Static anchoring at the initial router will be required. ii) No fixed IP address, i.e. the MU acts as client, but IP session continuity: No static anchoring at the initial router will be required. iii) No fixed IP address and no IP session continuity: no static anchoring at the Initial router will be required.

The rest of this paper is organized as follows. The paper first gives an overview of related concepts and initiatives within Open Networking Foundation (ONF) [11] and 3GPP [12] that are complement to S+ MN. Then introduces the S+ MN architecture, key features, interpretation of the internal functions with evolution of procedures of current mobile networks elaborated in SDN based mobile networks, analysis of current research towards IP mobility management and proposed improvements. In the next section it provides the functional validation and evaluation of proposed S+ MN architecture relative to a traditional architecture. The last section concludes the paper and discuss research directions.

\section{RELATED WORK}

This section provides an overview of concepts and initiatives with in ONF and 3GPP and that are relevant, extended or applied for S+ MN Architecture. This (.doc) document may be used as a template for papers prepared using Microsoft Word.

\subsection{SDN AND NETWORK VIRTUALIZATION}

SDN features various ways of using software to manage and manipulate network devices in which the control of gateway functions are lifted up to a logically centralized entity called SDN controller. The key concept of SDN is the separation of the control and data plane. It provides flexibility, high rate of innovation, and network programmability that speeds the deployment of new services. Figure 1 depicts a typical architecture of SDN. The data plane comprises a set of forwarding devices, (e.g., virtual switches or physical switches). The control plane consists of SDN controllers such as a Floodlight controller [13] or OpenDaylight controller [14]. The application plane consists of one or more applications, such as routing, and monitoring applications. The SDN applications communicate their network requirements towards the controllers via northbound interfaces such as REST API [15] or Java API [16]. The controllers communicate with each other using east/westbound interfaces. An open protocol is used to configure, monitor and manage forwarding devices through southbound interfaces. OpenFlow [17] protocol is the most extended SDN interface maintained by the Open Networking Foundation (ONF). The ONF has established the Wireless and Mobile Working Group to explore how the OpenFlow can adapt for use in mobile networks. In a solution brief [11] they illustrate the benefits of SDN/OpenFlow with use cases of inter-cell interference management, Mobile traffic management and envisioned that the SDN/OpenFlow benefits can also be realized throughout the mobile networks from access to backhaul and to the EPC. 


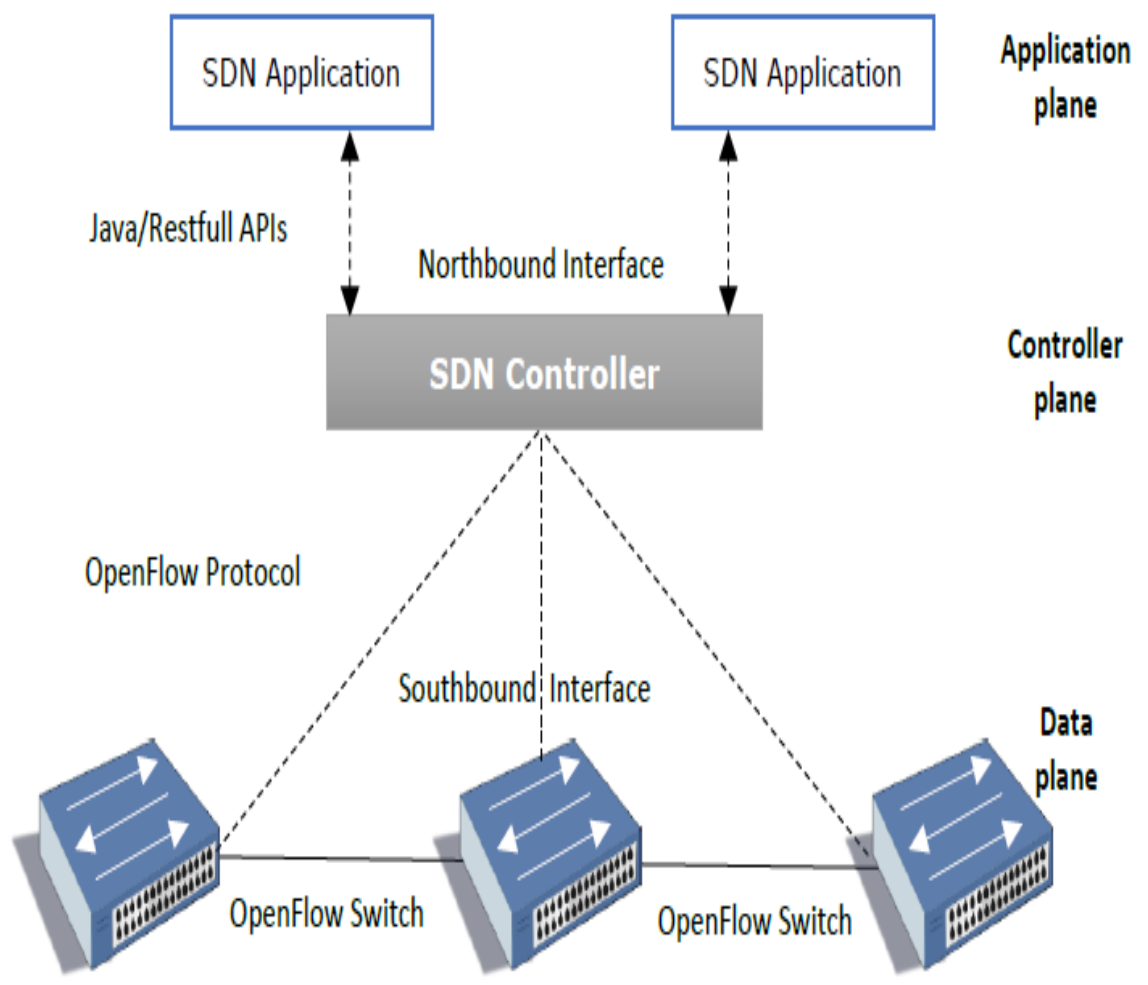

Figure 1. Typical Open SDN Architecture

Virtualization on the other hand is a useful technology that allows multiple users to share the same physical infrastructure by abstracting and decoupling the computing functionalities from underlying hardware [6]. During recent years virtualization technologies have moved from server and network domains to virtualization of mobile network functionalities. New applications of network virtualization can enable a more flexible management of network interconnections and can potentially enable different resources to coexist on the same virtualized infrastructure. There are different scopes and perspectives of mobile networks virtualization that are suitable for different applications and services and can be combined with configurable SDN technologies to address the challenges identified above in the current mobile network architecture. Both SDN and virtualization have related mechanisms and therefore could complement one another. Control and management for computing resources based on virtualization and software defined technologies has been extensively studied and for cloud computing application is being tested in major field trials. Using similar logic, recently, many research works have tried to evolve the traditional mobile network architecture using two of these concepts to deal with the challenges faced by mobile operators, such as the rapid growth of mobile traffic and new services. As the mobile medium is an ever-changing environment, there is a need for the virtualization techniques used in host virtualization to be modified or adapted when applied to mobile network technologies.

\subsection{INTER-SYSTEM INTER-NETWORKING}

Based on the actual 3GPP specifications [18], the 3GPP's CN exposes generic, IP-based interfaces towards the different non-3GPP access networks. With implied pre-registration to $3 \mathrm{GPP}$ over non-3GPP access, heterogeneous traffic is enabled to route through the common core network and subsequently, the EPC services are available to the users. There are several challenges associated with integration of different RATs such as the provision of a common authentication, authorization, and accounting (AAA) scheme, an end-to-end quality of service (QoS) and seamless handover etc. The QoS provided by the source and target access systems should be nearly identical to sustain the same communication experience and handover latency 
should be no more than a few milliseconds etc. 3GPP Technical Specification [19], a study on architecture for next generation networks with separation of control and data plane, specified in this context the architectural requirements, key issues and their solutions with functions descriptions. In this paper the inter-system handover of a MU from a 3GPP access network towards a non 3GPP access network when adopting SDN and virtualization in mobile networks and possible improvements for IP mobility management are considered.

\subsection{MULTIPATH TCP (MPTCP)}

MPTCP is an ongoing effort within Internet Engineering Task Force (IETF) to support multipath operation, a set of extensions to enables a regular TCP connection to use multiple different IP addresses and interfaces [20]. In the mobility context, when MU moves from one point of attachment (in 3GPP/EPS terminology PGW) to another i.e., it receives or configures a new IP address through new network attachment. MPTCP enable multiple IP addresses by adding subflows. Each subflow behaves as a separate regular TCP connection inside the network. Subflows can be added and removed at any point of time, in any MPTCP ongoing communication, with the help of ADD_ADDR option and REMOVE_ADDR option for any interface [20]. To maintain the ongoing communication MPTCP support "make before break" method and uses MP-PRIO option to specify any subflow as backup mode. In Figure 2, in the mobility scenario, With the MU have multiple IP addresses so in this case MPTCP can create multiple subflows and the MU is connected to Packet Data Network (PDN) service. Defined by MPPRIO option MPTCP support different flow modes, in the single-path mode only one TCP subflow is used at any time or using all subflows simultaneously between two communication nodes or uses only a subset of subflows for transmission of data packets. this paper explore the implied key role of transport layer protocols for IP mobility management by simultaneous exchange of IP traffic flows through the use of multiple distributed IP anchors.

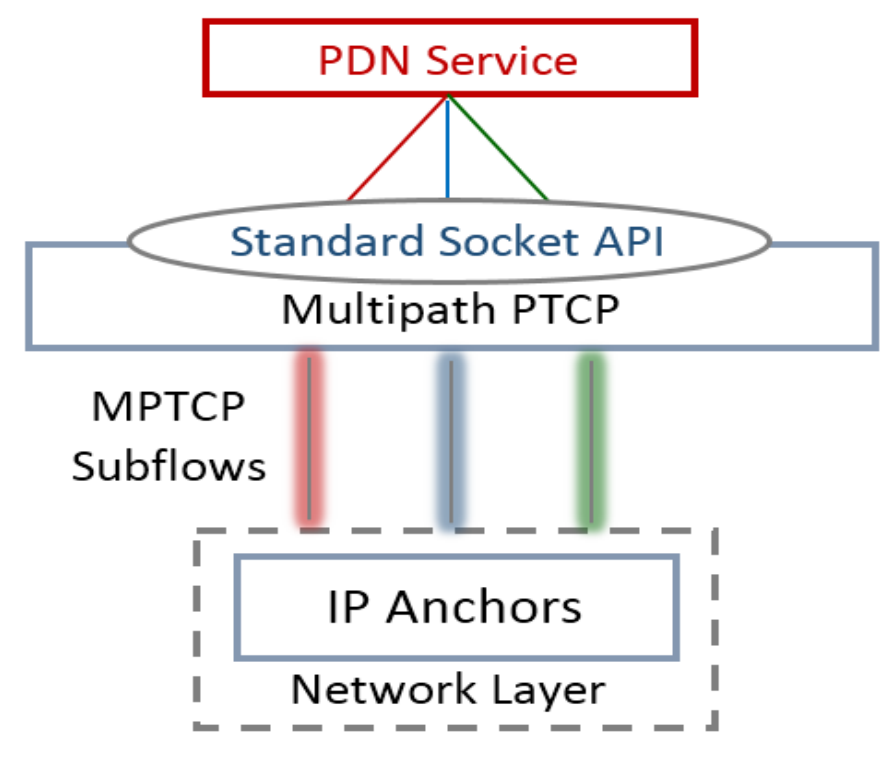

Figure 2. Multipath TCP multipath operations

\section{S+ Mobile Network ArChitecture}

Using the concepts of separation of control data plane, abstraction and sharing of resources we design an evolution of current mobile network architecture what we refer as Software defined plus virtualization featured Mobile Network $(\mathrm{S}+\mathrm{MN})$. Figure 3 shows the conceptual view of $\mathrm{S}+$ $\mathrm{MN}$ architecture, data plane enables the user traffic to be forwarded through the Access Network 
(AN) to core network gateways which terminate the traffic to external network. Software defined Plus virtualization featured Radio access network Controller (S+ AC) and Software defined Plus virtualization featured core Network Controller $(\mathrm{S}+\mathrm{NC}$ ) are proposed, the $\mathrm{S}+$ controllers $(\mathrm{S}+\mathrm{AC}$ and $\mathrm{S}+\mathrm{NC}$ ) can control the physical or virtual resources through programmable interfaces and have the ability to interact both access as well as forwarding nodes by consolidating redundant Access Stratum (AS) and Non-Access Stratum (NAS) signaling and management functions. The interpretation of the functions of the proposed architecture that is used to describe as follows:

The AN comprises the heterogeneous access environment that includes multiple different RATs links such as LTE (3GPP RAN), Wi-Fi (non 3GPP trusted RAN) and any new AN. The physical RAN resources can be abstracted and sliced into virtual RAN resources. In such case the new communication entity S+ Access Node can be presented that includes the collection of logical components of multiple different RATs nodes. The evolved access nodes directly interact with the physical radio resources and should enabled by the attributes of SDN (simple OpenFlow switch shall be added to these devices in order to support Open SDN protocols). The S+ AC shall act as a network administrator, coordination, policing and configuration of the infrastructure. The network control information is delivered from $\mathrm{S}+\mathrm{AC}$ to access nodes through the standardized southbound interface, i.e. OpenFlow. S+ AC shall apply access network-wide functions such admission control, radio resource scheduling, backhaul links management and QoS etc. In considered context to provide mobility support with Inter-system inter-networking be applied the MUs can obtain connectivity either from 3GPP or non-3GPP access node and move between them. Being the research focused on provision of IP session continuity during GW relocation and due to the paper length limitations, we cannot delve into details of S+ AN and mainly describe the realization of S+ core network.

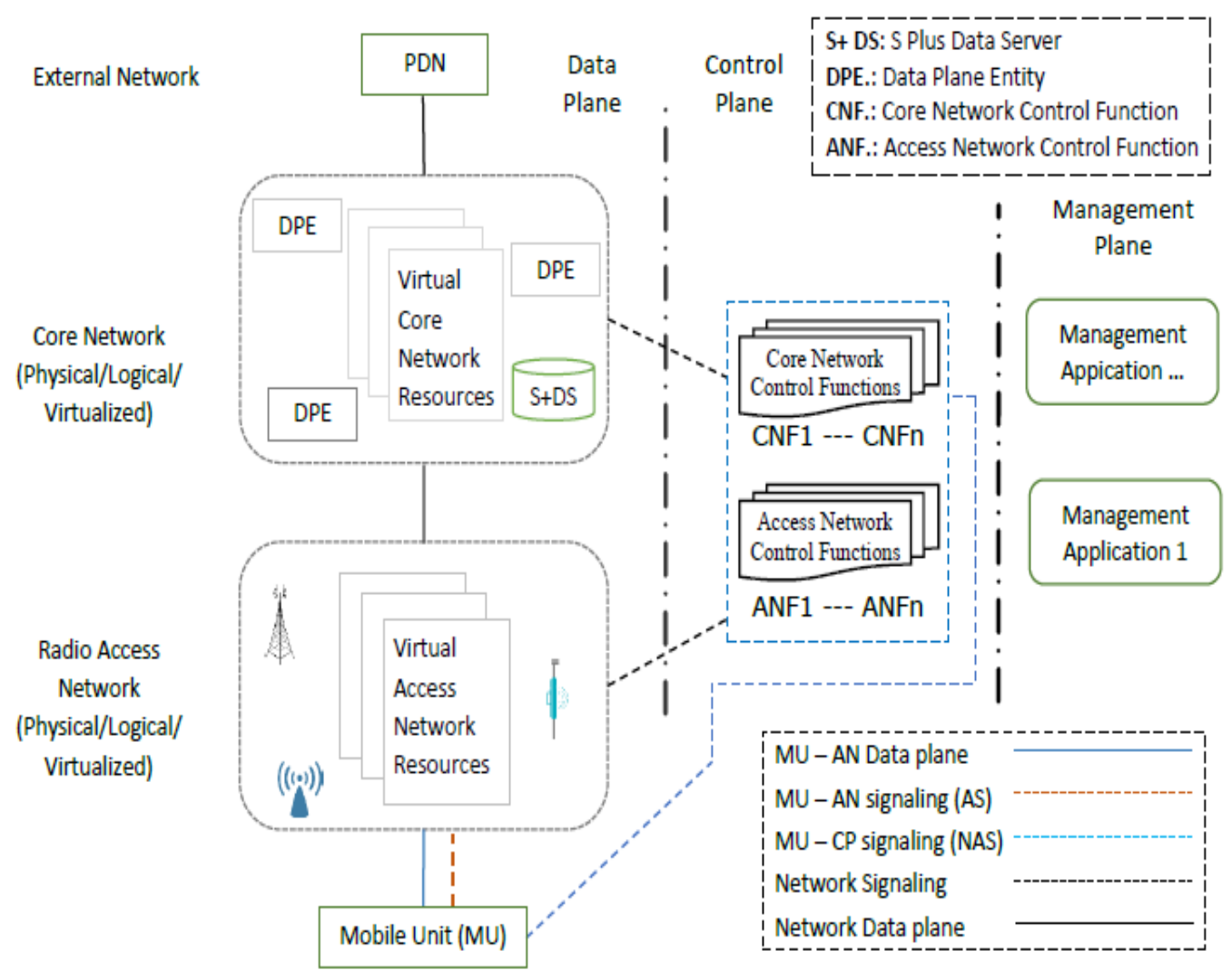

Figure 3. Conceptual view of S+ Mobile Network Architecture 
The Core network of S+ MN is composed of simple network gateways in contrast to legacy EPC, explicitly separate the data plane from control plane. Furthermore, it applied the distribution of gateway function. S+ NC manage the forwarding paths, calculates, installs rules into the user forwarding elements, path management, tunnel management, etc. In the following we provide details of S+ MN core network with evolution of procedure of current mobile networks elaborated with the separation of data and control plane.

\section{Realization Of S+ Mn Core Network}

The realization of S+ MN core network architecture is depicted in Figure 4, the S+ NC is the brain of the operations. All $\mathrm{CN}$ control functions are realized as set of control plane applications (using 5G terminologies Access and Mobility Management Functions (AMF), Session Management Function (SMF) and Policy Control Function (PCF) running on top of the S+ NC. The application uses the northbound interface to access the substrate from the S+ NC. Depending on the implementation of control plane and requirements of supported services these applications can have different functionalities. The AMF control function, can interact with subscription and content server named S+ DS to create a local copy for user authentication and authorization. For mobility management AMF tracks and updates the user's location information as well as their requirements. It delegates various event to $\mathrm{S}+$ access node such as initial context setup request and response for user attachment. AMF sends paging message to each access node belonging to the Tracking Area (TA) where the MU is registered and can also interact with SMF directly and PCF indirectly to support user session setup.

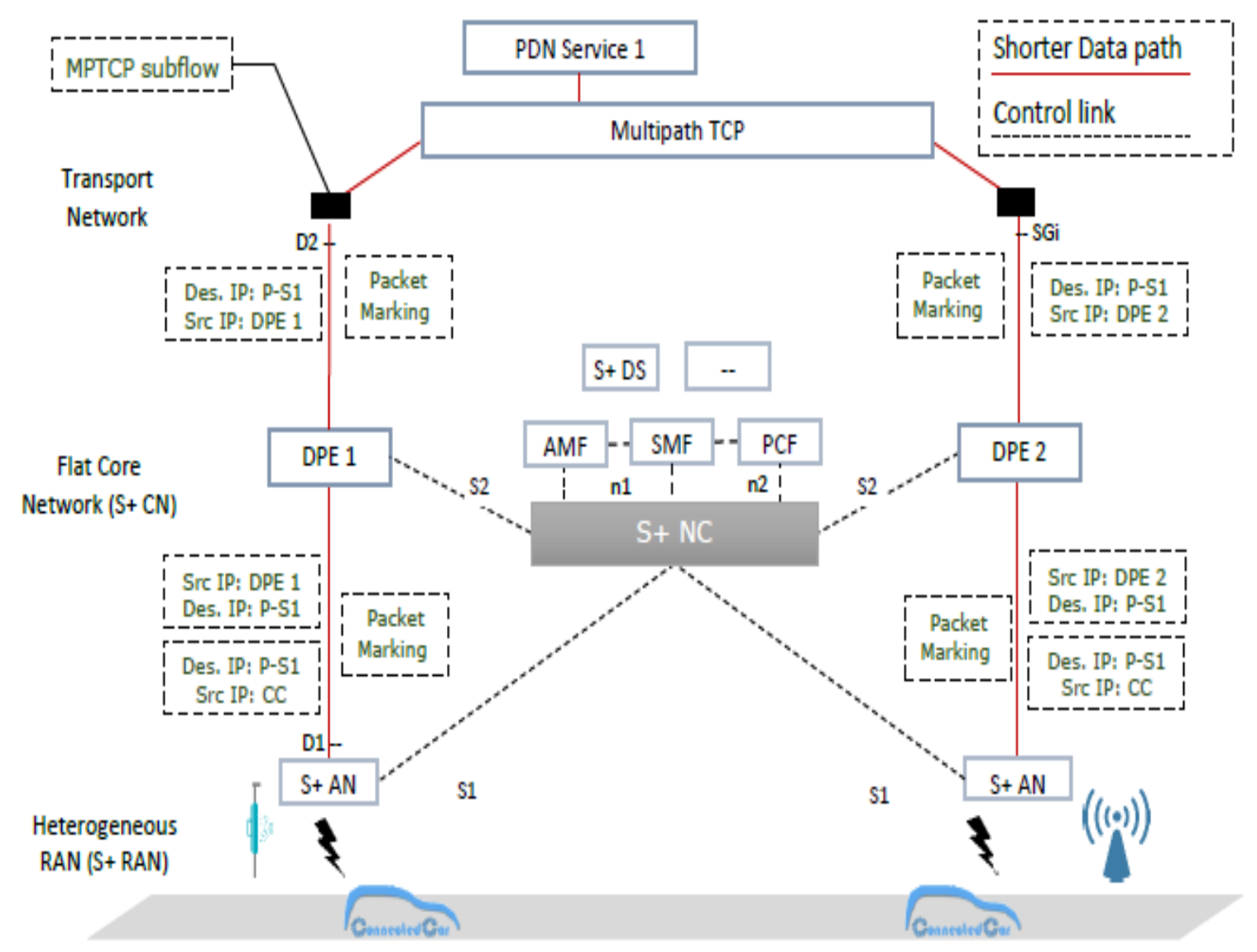

Figure 4. Conceptual view of S+ Mobile Network Architecture 
The SMF inherits the control functions performed by the PGW such as IP assignments, configuration of data plane path, selection and control of appropriate data plane entity, data plane management in a distributed manner, re-selection of DPEs, and QoS provisions etc. The SMF needs to interact with the PCF to get the corresponding policies in the case where the session requires a specific QoS policies. The AMF and SMF function will use a northbound interface (e.g., REST API) with the S+ NC which translates northbound messages into OpenFlow messages. When the flow rules are associated for subsequent packets that belong to the same section and installs them for the data plane. Furthermore, in order to support fully flexible IP flow mobility during DPE relocation AMF and SMF applications can have additional functionalities. Such as, provide distinct ID address for each flow. The default flow, if required, is anchored in the core at initial DPE with a fixed IP address of the user so that the user is always reachable for incoming requests. Other IP flows may be anchored with temporary IP addresses at local DPEs supporting session continuity when needed and decided upon by the user. For application oriented forwarding setup, change the forwarding rules of the data plane switches for a specific flows or for all flows of the handled MU.

Distribution of gateway function approach has been followed to avoid unnecessary longer data path and evolved with SDN technology. Using 3GPP terminology each DPE implements Data Plane Serving Gateway (D-SGW) or Data plane PDN Gateway (D-PGW) functions and communicates with an S+ NC through OpenFlow protocol that implements the southbound interface. DPEs use the flow rules received from the S+ NC and have standard mobile network tunnel processing capabilities with a GTP-U extension (e.g., encapsulation/decapsulation facilities) and forward packets to the transport network. Data plane may also include the key functionality of a wireless access node, such as interfacing to manage mapping between QoS flows and Data radio bearers (DRBs). During an inter system handover or DPE relocation, the S+ NC needs to send OpenFlow Packet Out messages to the source as well as target DPE in order to modify their flow tables at the same time.

\section{Evaluation Methodology}

We discuss a scenario used to explain the behavior is the inter-system handover of a MU from a 3GPP access network towards a non 3GPP access network and describe the important call control flows being exchanged across the various network elements of the proposed architecture to show how it can be systematically exploited to gain benefits:

\subsection{Initial Attachment Procedure}

Figure 5 shows the signalling diagram of initial attachment, illustrating an expression of proposed architecture. The 3GPP/LTE compliant evolved access node S+ eNB receives an attachment request message (1) from MU. This message is embedded in an OpenFlow Initial User message (2) and is sent to the $\mathrm{S}+\mathrm{NC}$, where the proposed AMF application initiates MU authentication and fetches users' subscription profile from S+ DS. After the successful authentication and authorization, the OpenFlow Initial Context Setup Request message (3) is sent from the S+ NC to the $\mathrm{S}+\mathrm{eNB}$. The $\mathrm{S}+\mathrm{eNB}$ reconfigures the radio connection, and forward the attach accept message to the MU. The acknowledgment of attach complete from MU is included by S+ eNB as context setup response message (4) to the controller. The state of MU transitions from deregistered to registered and transmits the first session packet to the S+ eNB over the radio connection. Since, there is no matching flow entry in the flow table of the $\mathrm{S}+\mathrm{eNB}$. The $\mathrm{S}+\mathrm{eNB}$ triggers an OpenFlow packet-in message (5) to the $\mathrm{S}+\mathrm{NC}$. This message includes some information that is necessary to establish the data plane. The S+ NC analyse the packet header to obtain the session information such as the source and destination nodes IP addresses and collects the transport marking information. S+ NC assign the DPE by interacting with the SMF applications. In turn the MU obtain an IP address from SMF, this also includes as conferred in 
[21] creating Binding Cache Entry (BCE) to keep track the MU's location as well as the related information. Then, the S+ NC creates flow rules for subsequent packets that belong to the same section and send them as OpenFlow packet-out message (6) to installs them for the S+ eNB and DPE. In the case where the session requires a specific QoS policy, the S+ NC needs to interact with the PCF to get the corresponding policies before downloading the flow rules to the data plane. When the flow rules are associated with a QoS parameter, the S+ NC will install them at the DPE. Furthermore, considering the expected handover with MU mobility, The AMF in cooperation with the SMF notify the needed support for DPE relocation and keep the established PDN-Connection context. At the end of this procedure, the MU state is transitioned from Idle to Connected and a data forwarding path is established from the MU to the correspondent PDN service.

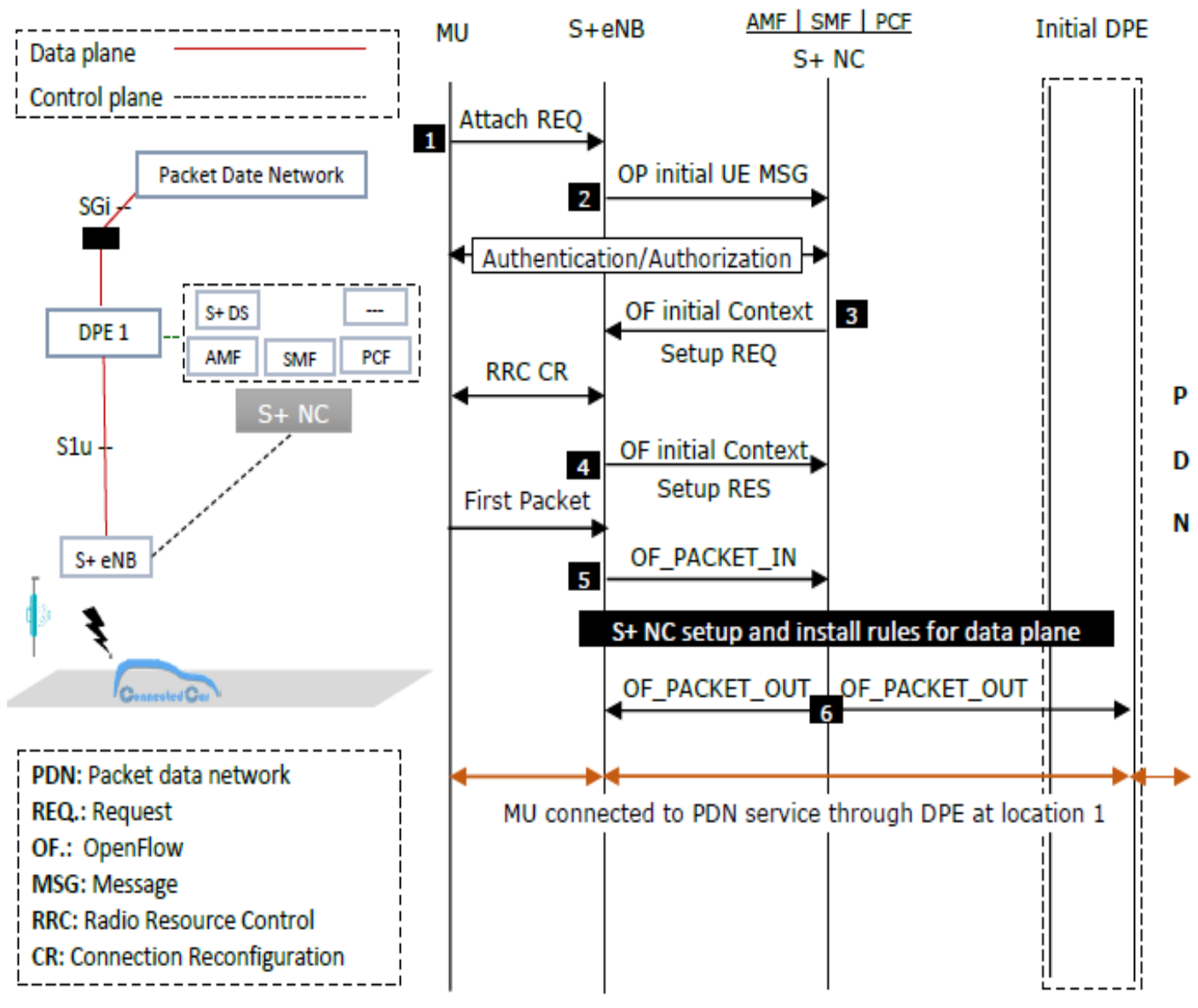

Figure 5. Initial attachment operation

\subsection{INTER-SYSTEM HANDOVER}

As shown in Figure 6, the MU is initially connected to 3GPP access network, when the MU moves away from source S+ eNB and determine the presence of appropriate access node (in this case a non-3gPP AP), S+ eNB sends a Handover required message packaged in an OpenFlow message to the $\mathrm{S}+\mathrm{NC}(1)$. The AMF function in turn trigger the handover by sending a Handover Request message (2) to the target non-3GPP compliment access node (S+ AP). If targeted S+ AP response with handover ACK message (3) to the S+ NC, the handover command (4) is send toward the MU by $\mathrm{S}+\mathrm{NC}$ and then MU trigger the attachment procedure (Access technology specific procedure for the interworking of the 3GPP and non-3GPP access networks) [18]. After 
attachment $\mathrm{S}+\mathrm{NC}$ receives packet-in message (5) from $\mathrm{S}+\mathrm{AP}$ and it must connect a set of appropriate DPE that is close to the MU. Together with this procedure MU gets a new IP address to be used in the new flow. S+ NC allocates new IP address to MU and have to establish a data forwarding path according to application needs.

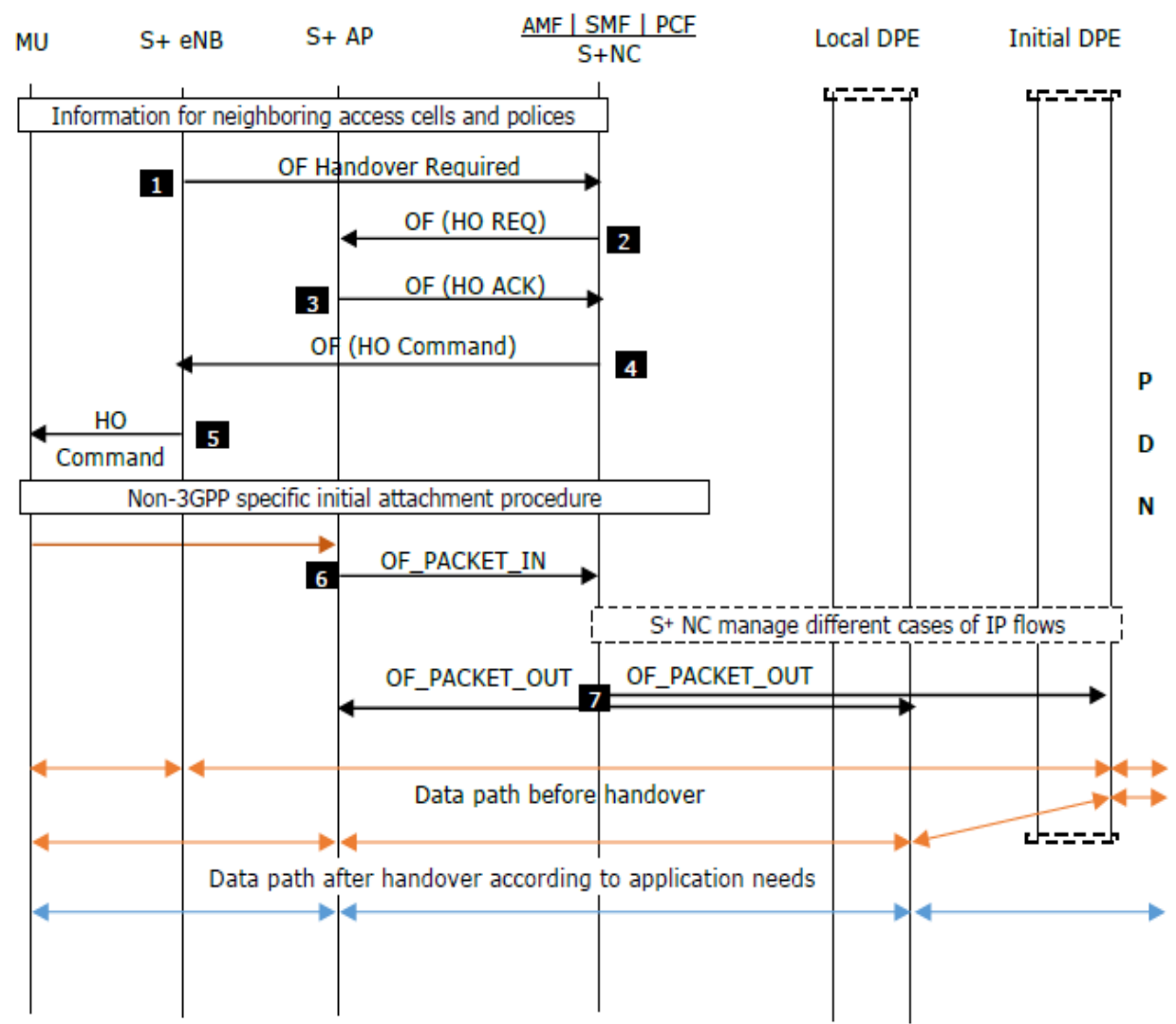

Figure 6. S+ MN based inter-system handover

\subsection{INTER-SYSTEM HANDOVER}

There have been several approaches for IP mobility management in SDN based mobile networks, as surveyed in [21, 22], In, [21] the author applied the SDN concept to DMM architecture for routing optimization with a DMM service within SDN controller. In proposed architecture, when a user attach initiate router, the SDN controller stored user information in Binding cache entry (BCE). If the user moves to the new router, the controller which receives packet-in message will check BCE. Mobility management is supported to user by binding update with DMM service. The new data path is set by SDN controller and it sends Flow Modify message to previous and new router. On receiving flow modify messages, the routers will update their flow tables. That is, user can be supported optimized path by flow table, without tunneling. But It is noted that after handover, first, the packets have to follow the route from the previous to the new router. The packets are then redirected between the new router and fInally to the user. Consequently, it may lead to a complex process and a high signaling cost.

In [22], the author considered two schemes for mobility management in DMM scenario called Tunneling mode and Optimal mode DMM, in first scheme different prefixes are allocated to user at old and each new attachment nodes. For the ongoing flows which require mobility support, the list of old and new prefixes are included in packet-out message named router advertisement message. The controller updates the forwarding rules at both old and new OpenFlow switches (OFSs). Using tunneling mechanism the flows are being redirected between distributed OFSs. In 
second scheme, the controller calculates the new route for the on-going flows and populates the new forwarding rules to all the intermediate OFSs along the new route between the new OFS and the Correspondent Node $(\mathrm{CN})$. In other words, an explicit path between the user node and $\mathrm{CN}$ is defined and established by the controller. In this way, tunneling mechanism can be avoided. However, it may lead to a complex task giving the large number of switches/flows to handle. Besides, the simplicity of the first scheme can come at the cost of tunneling overhead and suboptimal routing. the authors indicated that the optimal mode is likely more suitable for latency sensitive services while the tunnel mode seems to be better for the packet loss-and interruptionsensitive services.

To fully appreciate these particular challenges associated with IP mobility, the proposed architecture implied MPTCP at transport layer. Since MPTCP does not change the socket API, it can be used transparently by any PDN service and change of IP address of a MU does not force the connection to be restarted. A combination of SDN capabilities and MPTCP options could be used to remove the chains of IP preservation of current mobility management solutions and disburden the process of flow forwarding during DPE relocation. With implied MPTCP connection of MPTCP capable MU and PDN-Service and will be able to synchronize the MU traffic using different IP addresses. As shown in figure 7, the S+ NC can manage different cases of IP flows, the data packets from service requiring static IP anchoring are directed to initial flow, so the MU is enabled to keep the initially assigned IP address despite its location changes and where no static anchoring is required, the MU uses the new flow for active communication, while maintaining the reachability for the IP address that is still in use. In this way the need for the maintaining a tunnel between source and target anchors and flow redirection is not required to link different flows, leading in turn to seamlessly session continuity during DPEs relocation avoiding a large number of signalling messages. The S+ NC monitor the MU activity, as no traffic is carried in the initial subflow (during the timer interval) it starts the releases procedure for the removal of initial IP address from the PDN-S1 IP list. The release cause tag along the session management and perform the DPE binding information update.

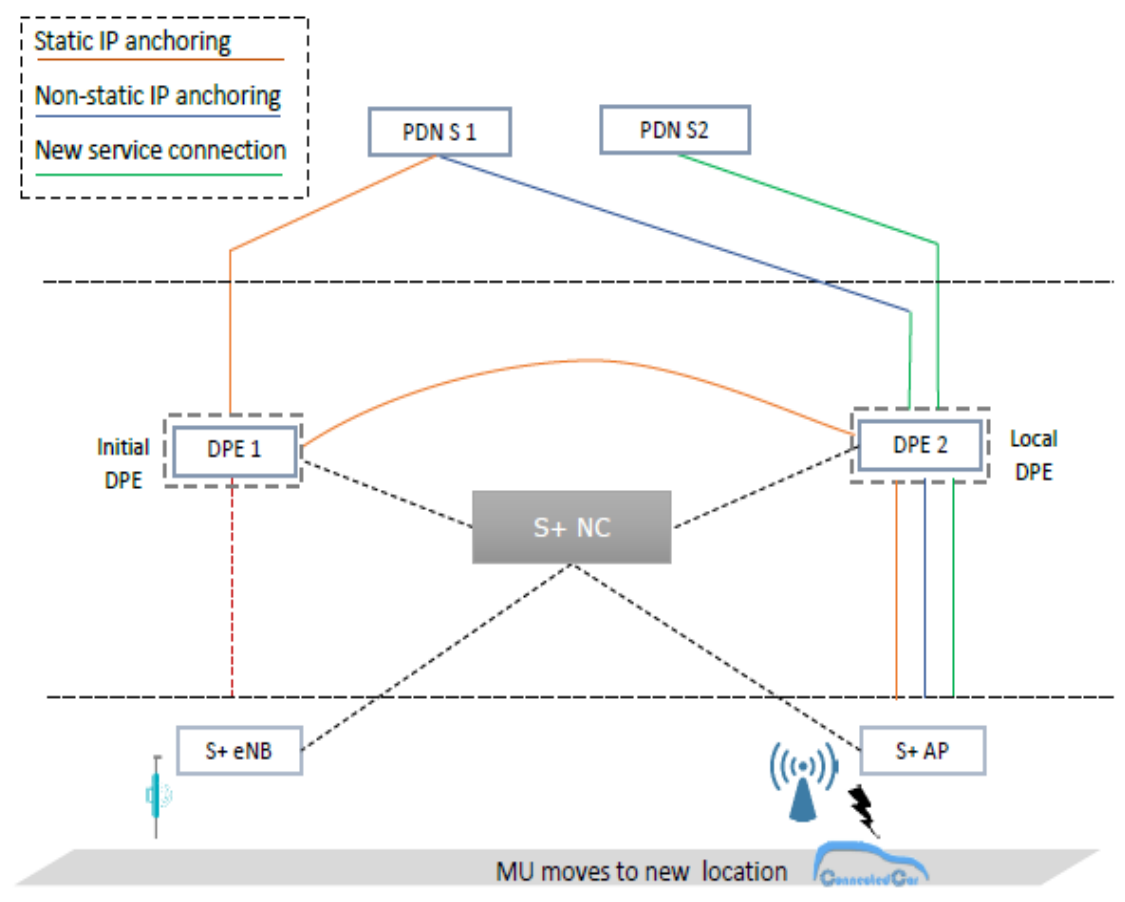

Figure 7. S Flow setup to application needs 


\section{FUnCTIONAL VALIDATION}

To evaluate the working and key benefits of the proposed S+ Mobile Network architecture, we developed an ns-3-based system level simulation test setup, which primarily consists of point to point helper, network, applications, mobility and core modules. Besides this, OFSwitch13 module [23] has been implemented in ns-3 simulator that enable support for OpenFlow protocol version 1.3. we extended the NS3 environment to implement instantiation of multiple DPEs and modified the currently deployed X2-based handover procedure of the NS3 to support relocation of DPE during the MUs handover. It implements the messages exchange between the different network functions for inter-system handover and DPE relocation enforcing the policies and the behaviour described in section 5.1 and 5.2. The source code of implemented modules is available via [24]. The logical network topology of the evaluation scenario is shown in Figure 4. The simulation starts with a MU initially attached to the PDN service 1 via 3GPP compliant access network. Moving out of S+ eNB coverage area the MU discovers and switches to non-3GPP compliant access connection. We set the parameters for simulation in which data packets are set to 1000 units and simulation interval is $10 \mathrm{~ms}$. For each handover case, we measured the preparation, interruption and completion time. Figures $8 \mathrm{a}$ and $8 \mathrm{~b}$ depicts the measured handover execution time, whose estimated latency values were around $5.7 \mathrm{~ms}$ and $7.47 \mathrm{~ms}$, respectively. These results fulfil the control plane delay requirements of the $5 \mathrm{G}$ networks.

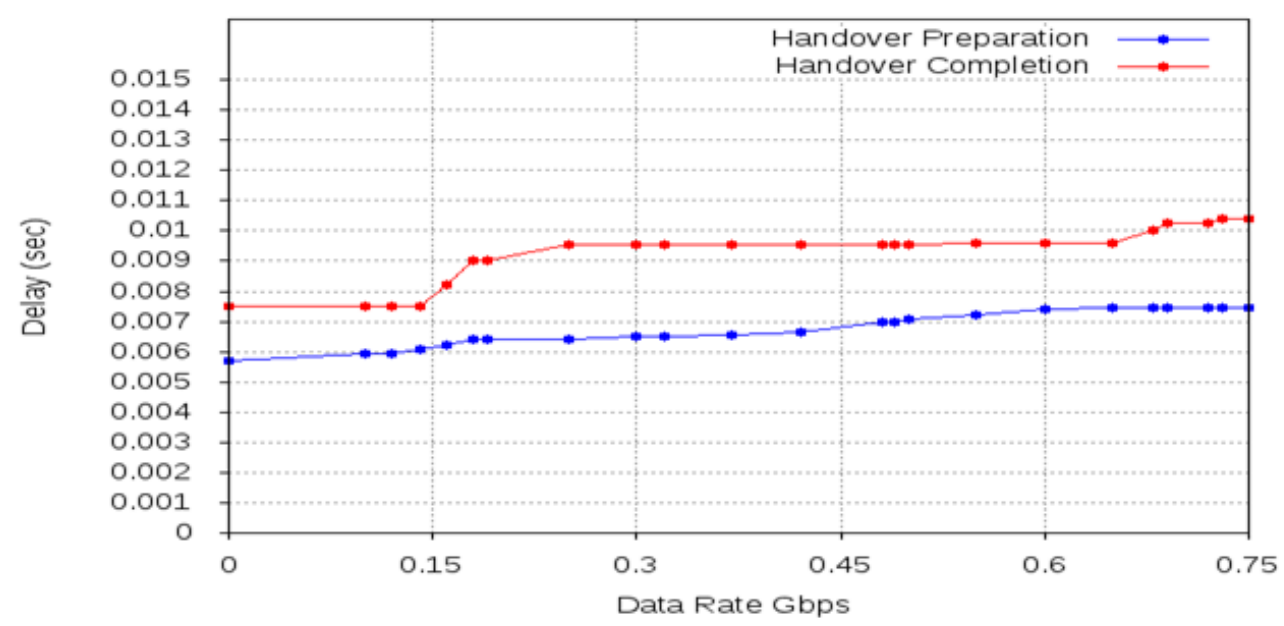

Figure 8a. Inter-system handover execution time

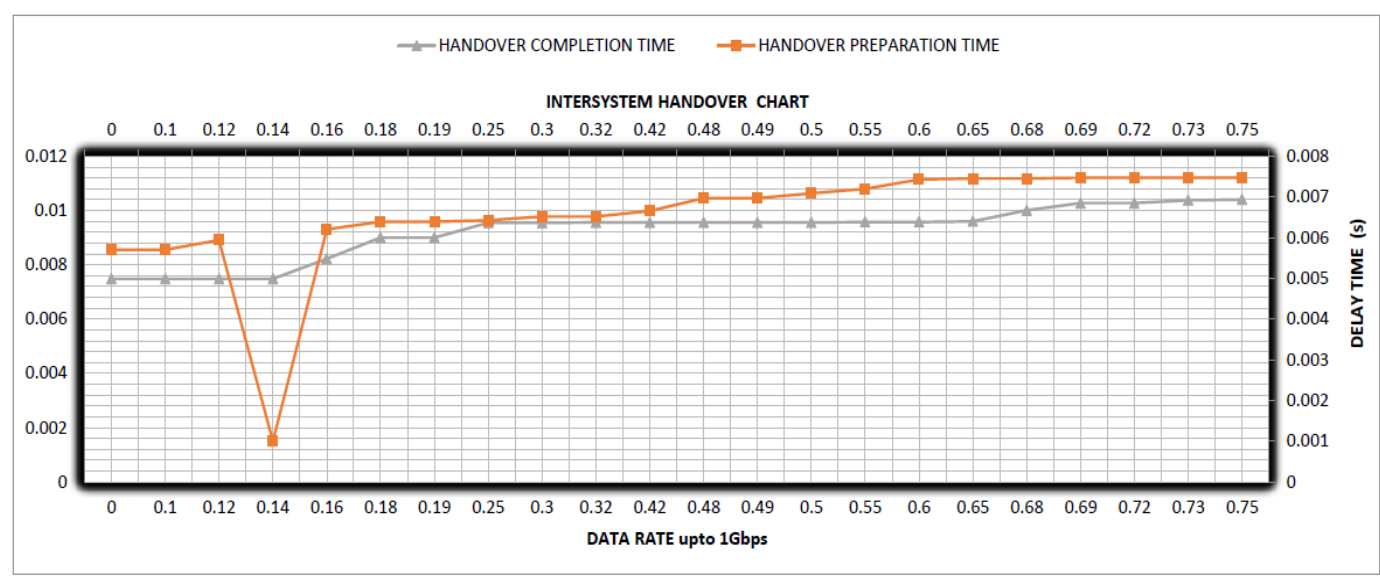

Figure 8b. Inter-system handover measurements 


\section{Conclusions}

This paper presented evolution of the mobile network architecture in SDN and virtualization context and provided improvements for DMM to enable seamless handover while roaming across heterogeneous access networks, novel perspective to maintain session continuity during the movement of MU that tag along with DPE relocation. The MPTCP protocol is implied to remove the chains of IP preservation of current mobility management solutions, leading in turn to seamlessly change the IP address during a connection without breaking and re-establishing the connection. A functional validation setup has been devolved to show the working of proposed improvements. The pictorial call control flows thus created has managed different cases of IP flows and shown a significant decrease in signalling and delay compared to tunnel and routing based approaches of IP mobility management. The simulation results show that the proposed evolutions efficiently fulfill the functionality and performance requirements (e.g., seamless mobility management, setting up traffic redirection path and maximum allowed delay) during inter-system handover. In future work we intend to perform more detailed experiment with MPTCP to illustrate further system realization of the architecture presented.

\section{REFERENCES}

[1] Index, C., Cisco Visual Networking Index: Global Mobile Data Traffic Forecast Update, 2016-2021. 2017.

[2] 3GPP, TS 36.300, Evolved Universal Terrestrial Radio Access (E-UTRA) and Evolved Universal Terrestrial Radio Access Network (E-UTRAN). 2018.

[3] H. Chan, D.L., P. Seite, H. Yokota, J. Korhonen, , IETF RFC 7333 "Requirements for DMM 2014.

[4] Dawood, M., F. Woldemar, and B. Ghita, Assay of Multipath TCP for Session Continuity in Distributed Mobility Management., in Proceedings of the Eleventh International Network Conference (INC 2016) Frankfurt am Main Germany. 2016.

[5] Nguyen, V.-G., T.-X. Do, and Y. Kim, SDN and Virtualization-Based LTE Mobile Network Architectures: A Comprehensive Survey. Wireless Personal Communications, 2016. 86(3): p. 14011438.

[6] Barona López, L.I.; Valdivieso Caraguay, Á.L.; Sotelo Monge, M.A.; García Villalba, L.J. Key Technologies in the Context of Future Networks: Operational and Management Requirements. Future Internet 2017, 9, 1

[7] Karimzadeh, M., L. Valtulina, and G. Karagiannis, Applying sdn/openflow in virtualized lte to support distributed mobility management $(\mathrm{dmm})$, in The 4th international conference on cloud computing and services science (CLOSER) 2014. p. 1-6.

[8] Sama, M.R., et al. Enabling network programmability in LTE/EPC architecture using OpenFlow. in Modeling and Optimization in Mobile, Ad Hoc, and Wireless Networks (WiOpt), 2014 12th International Symposium on. 2014.

[9] Yaz, V., U.C. Kozat, and M.O. Sunay, A new control plane for 5G network architecture with a case study on unified handoff, mobility, and routing management. IEEE Comm. Magazine, 2014. 52(11): p. $76-85$.

[10] Sama, M.R., et al., Software-defined control of the virtualized mobile packet core. IEEE Communications Magazine, 2015. 53(2): p. 107-115.

[11] ONF, OpenFlow Enabled Mobile and Wireless Networks, ONF Solution Brief. 2013.

[12] 3GPP Scope and Objectives. 2017; Available from: http://www.3gpp.org/

[13] Project Floodligt: an Open SDN Controller. Available from: http://www.projectfloodlight.org/floodlight/

[14] OpenDaylight: Open Source SDN Platform. Available from: https://www.opendaylight.org/ 
[15] Richardson, L., M. Amundsen, and S. Ruby, RESTful Web APIs. 2013: O'Reilly Media, Inc. 408.

[16] Java API Specifications. Available from: http://www.oracle.com/technetwork/java/api-141528.html

[17] ONF, TS-020: OpenFlow switch specification version 1.5.1.

[18] 3GPP, TS 23.402 Architecture enhancements for non-3GPP accesses. 2016.

[19] 3GPP, TS 23 799; Study on Architecture for Next Generation System. 2016.

[20] Ford, A., et al., TCP Extensions for multipath operation with multiple addresses, in RFC 6824, IETF. 2013.

[21] Hyunsik Yang, K.S., Younghan Kim, IETF RFC: Routing Optimization with SDN.

[22] Nguyen, T.T., C. Bonnet, and J. Harri. SDN-based distributed mobility management for 5G networks. in 2016 IEEE Wireless Communications and Networking Conference. 2016.

[23] OpenFlow 1.3 module for Ns-3. Available from; http://www.lrc.ic.unicamp.br/ofswitch13/

[24] Dawood, M. and M.S. Shafi. S plus mobile network archtecture implementation. 2018; Available from: https://github.com/mshary/S-plus-Mobilhe-Network.

\section{AUTHORS}

Muhammad Dawood (muhammad.dawood@plymouth.ac.uk) received his master's degree in information technology from university of applied sciences Fulda, Germany. Since 2013 he is researcher at Centre for Security, Communications and Network Research, University of Plymouth, UK where he is $\mathrm{PhD}$ student in future mobile systems architecture. His current research focus on software defined plus Virtualization featured Mobile Network architecture for vehicular communications.

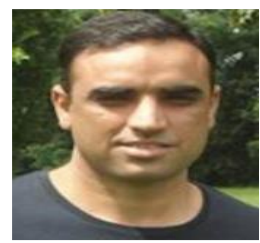

Woldemar Fuhrmann (w.fuhrmann@fbi.h-da.de) is Professor in computer sciences and telecommunications at university of applied sciences Darmstadt, Germany. He is also director of studies for advance research in telecommunication systems at Centre for Security, Communications and Network Research (http://cscan.org/?page=staffprofile\&id=13), University of Plymouth, UK. His major areas of research include mobile communication systems, machine type communications, and the internet of things.

Bogdan V Ghita (bogdan.ghita@plymouth.ac.uk) is an Associate Professor (Senior Lecturer) in Computer Networks at the University of Plymouth, UK. From the same university he received his Ph.D. (2004) degrees in Performance characterisation of IP Networks. His major areas of research include QoS measurement and provisioning, user and application profiling and fairness.
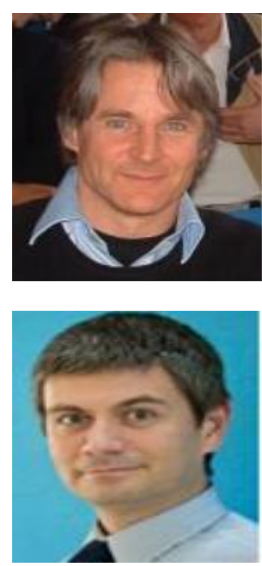